Bedeutung. Der Muskel ist kein, vom Sternomastoideus der entsprechenden Seite etwa abgezweigtes Bündel, da letzterer Muskel ja in seiner vollen Entwickelung zugegen ist. Der Muskel ist ein selbständiger accidenteller Muskel, ron anderen Tensores fasciae colli, mögen diese nun Abzweigungen von anderen Halsmuskeln, oder selbständige, von anderen Orten ausgegangene Muskeln sein, völlig verschieden, wie ich schon 1872 behauptet habe. Er gleicht dem Muskel des früheren Falles, abgesehen von dem breitsehnigen und weiter nach auswärts und zwar bis hinter und lateralwärts von dem Sternomastoideus sich erstreckenden Ursprunge des letzteren und von anderen unwesentlichen Eigenschaften.

Ich habe den neuen Fall mitgetheilt, um darzuthun, dass auch dieser anomale Muskel öfter auftreten könne.

Das Präparat habe ich in meiner Sammlung aufbewahrt.

\title{
IV. (CLXXXIV.) In Bildungshemmnng begründetes, anscheinend bis über den 1. Lendenwirbel verlängertes und mit einem Ramus communicans vor dem 5 . Lendenwirbel rersehenes Auftreten der Venae iliacae communes. \\ (3. Fall eigener Beobachtung.)
}

Zur Beobachtung gekommen am 13. September 1880 an der Leiche eines Mannes. Das Präparat habe ich gleichfalls in meiner Sammlung aufbewahrt.

Der Fall gleicht in Manchem dem Falle, welchen ich in "No. CLVIII meiner anatomischen Notizen " ${ }^{1}$ ) beschrieben und daselbst mit den bis dahin gekannten Fällen ${ }^{2}$ ) verglichen habe, ist aber in Anderem auch davon verschieden, wie sich aus Nachstehendem ergeben wird:

Die aus der Vereínigung der Vena iliaca externa et interna jeder Seite in der Höhe des V. Lendenwirbels, hinter der Theilung jeder Arteria iliaca communis in ihre Aeste, entstandenen, und wie abnorm verlängerte, vor der Wirbelsäule in der ganzen Höhe ibres Lendentheiles aufsteigende Venae iliacae communes erscheinenden Venenstämme erstrecken sich diesmal weiter, und zwar bis vor das Lig. intervertebrale zwischen dem letzten Brust- und dem ersten Lendenwirbel, nach oben.

1) Dieses Archiv Bd. 81. 1880. S. 465.

$\left.{ }^{2}\right)$ Hierher gehört auch noch ein Fall von J. Hyrtl. Lehrb. d. Anatomie d. Menschen. Wien 1859. S.882. 
Zwischen beiden existirt auch ein Ramus communicans, aber dieser geht schon vom Anfange des linken Venenstammes und theilweise von dessen V. iliaca interna ab und mündet in den rechten Venenstamm schon 1,0 Cm. über der Vereinigung seiner V. iliaca externa et interna. Er verläuft daher schon vor dem V. Lendenwirbel, und zwar fast quer vor dessen Mitte von links nach rechts. Die beiden Venenstämme mit ihrem Ramus communicans begrenzen deshalb diesmal einen sehr langen, dreischenkligen Ring, welcher seinen unteren, geradlinigen kurzen Schenkel vor dem V. Lendenwirbel, seinen oberen, zugespitzten Winkel vor dem Lig. intervertebrale zwischen dem letzten Brust- und ersten Lendenwirbel, rechts von der Aorta abdominalis, liegen hat und ein $15,8 \mathrm{~cm}$. hohes und ganz unten $5,3 \mathrm{~cm}$. breites Lumen besitzt.

In diesem Ringe steigt die Aorta abdominalis, nachdem sie in der Höhe des I. Lendenwirbels den linken Venenstamm von hinten gekreuzt bat, allerdings ebenfalls, und zwar vom rechten Venenstamme in einer Distanz von bis $2,3 \mathrm{Cm}$., von der verticalen, seitlich von ihr gelagerten Portion des linken Venenstammes in einer Distanz von bis $1,3 \mathrm{Cm}$, etwas schräg nach rechts herab, aber die Stelle ihrer Bifurcation in die Arteriae iliacae communes vor der Mitte des IV. Lendenwirbels liegt diesmäl nicht sogleich, sondern in einer Distanz von $4,5 \mathrm{Cm}$, über dem Ramus communicans beider Venenstämme.

Der rechte Venenstamm nimmt ausser dem genannten Ramus communicans und ausser den Venae lumbales der entsprechenden Seite ungewöhnlich tief unten die V. spermatica interna dextra und, unter seiner Zusammenmündung mit dem linken Venenstamme in das kurze, abwärts von der Leber gelagerte Stück der V. cava inferior, die $V$. renalis dextra auf. Dieser 5 tamm ist $17,5 \mathrm{Cm}$. lang und $1,4 \mathrm{Cm}$. weit.

Der linke Venenstamm steigt bis oberhalb des If. Lendenwirbels seitlich von der Aorta abdominalis, dann schräg vor ihr unter dem Ursprunge der Arteria mesenterica superior, nach rechts und oben hinüber. Er nimmt, ausser Venae lumbales der entsprechenden Seite, bevor er sich mit der Aorta kreuzt, die an ibrer Mündung 1,4 Cm. weite V. renalis sinistra und, 1,2 Cm. höher vor der Aorta, an seiner schrägen Portion die Vena suprarenalis sinistra auf. Dieser Stamm ist $19,5 \mathrm{~cm}$. lang, woron auf seine obere schräge Portion etwa $\frac{1}{4}$ seiner Länge kommt. Bis zur Aufnahme der $V$. renalis ist er $1,4 \mathrm{Cm}$, also so wie der rechte Stamm, darauf aber $1,6 \mathrm{~cm}$, weit.

Nach dem Zusammenflusse beider Venenstamme ist die Vena cava inferior bis zur Leber diesmal nur $6 \mathrm{Cm}$. lang und $2,3 \mathrm{~cm}$. weit.

Die V. suprarenalis dextra ergiesst sich in die Vena cava inferior, die V. spermatica interna sinistra in die $\mathrm{V}$. renalis sinistra, $7 \mathrm{Mm}$. auswärts von deren Mündung in den linken Venenstamm.

Der Ramus communicans der Venenstämme, welcher hinter der Theilung der A. iliaca communis sinistra vom linken Stamme beginnt und in kurzer Strecke über der Theilang der A. iliaca communis dextra, die wie die Arterie der linken Seite $6 \mathrm{Cm}$. lang ist, hinter derselben in den rechten Venenstamm mündet, nimmt, ausser einem Paar kleiner, in ihn an seinem oberen Umfange mündender Venen, an seiner rechten Hälfte und am unteren Umfange, nabe der Medianlinie, die v. sacralis 
media auf. Am hinteren und unteren Umfange seines 0stium in den rechten Venenstamm sitzt eine mit ibrem Sinus gegen diesen offene Valvula, die auch in meinem II. Falle bei nachträglícher Untersuchung sich vorfand. Derselbe ist $5,3 \mathrm{Cm}$. lang, am rechten Ende $11 \mathrm{Mm}$., an der Mitte 5-6 Mm. und am linken Ende $9 \mathrm{Mm}$, weit.

Die Vena azygos und V. bemiazygos waren wie gewöhnlich angeordnet. Auch der Körper war übrigens wohl gebaut.

In diesem Falle einer, wie bekannt, in Bildungshemmung begründeten Venenanomalie, bei welcher der rechtsseitige Venenstamm, rom Ramus sommunicans angefangen bis zum Zusammenflusse mit dem linken Venenstamme, als unteres grösstes, aber etwas verengertes Stück der Vena cava inferior, der linksseitige Venenstamm bis zur V. renalis sinistra aufwärts als unterer erweiterter Theil der V. cardinalis sinistra und der Ramus communicans als verkümmerte V. iliaca communis sinistra aufzufassen ist, war das der V. iliaca communis dextra entsprechende Venenstück sehr kurz (1 Gm.), wie wohl noch in keinem der beobachteten Fälle. Die Theflung der V. cava inferior in die V. iliacae communes musste daher vom Ursprunge an abnorm tief unten vor sich gegangen sein und daher die zum Ramus communicans verkümmerte $V$. iliaca communis sinistra weit abwärts von der entsprechenden Arterie ihre Lage eingenommen haben, was die ungewöhnliche Form und die grosse Länge des von der Venenanomalie gebildeten Ringes bedingte. Der Fall ist der dritte der mir zur Beobachtung gekommenen Fälle dieser Venenanomalie, welcher mir schon 7 Monate nach dem letzten Falle vorkam, während ich die beiden ersten Fälle erst in einem Zwischenzeitraıme von 20 Jahren angetroffen hatte.

\section{V. (CLXXXV.) Zergliederung des Doppeldanmens beider Hände einer Fran.}

(6. und 7. der zergliederten und 10. und 11. der überhaupt untersuchten Fälle an Erwachsenen nach eigener Beobachtung.)

(Hierzu Taf. XX.)

Vorhanden an beiden Händen einer über 80 Jahre alten Frau. Zur Zergllederung am 7. April 1881 erhalten. Die Zergliederung nach vorausgeschichter Injection der Arterien vorgenommen. Beide zergliederte Hände in meiner Sammlung aufgestellt.

Die Vorderarme und die Hände, letztere selbst abgesehen von den Daumen, sind für eine Frau zu breit und zu stark. Die Daumen der rechten Hand (Fig. 1) 